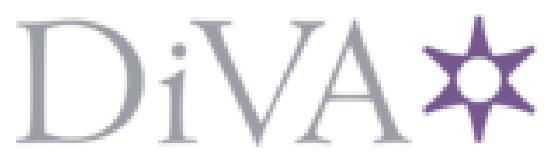

http://www.diva-portal.org

This is the published version of a paper presented at 21st European Conference on Power Electronics and Applications (EPE' 19 ECCE Europe), Genova, Italy.

Citation for the original published paper:

Nahalparvari, M., Karamanakos, P., Geyer, T. (2019)

Gradient-Based Fixed Switching Frequency Direct Model Predictive Control for Grid-

Connected Converters with LCL Filters

In: Proceedings of the IEEE European Conference on Power Electronics and Applications, Genova, Italy, Sept. 2019 Institute of Electrical and Electronics Engineers (IEEE)

https://doi.org/10.23919/EPE.2019.8915403

N.B. When citing this work, cite the original published paper.

Permanent link to this version:

http://urn.kb.se/resolve?urn=urn:nbn:se:kth:diva-264968 


\title{
Gradient-Based Fixed Switching Frequency Direct Model Predictive Control for Grid-Connected Converters with $L C L$ Filters
}

\author{
Mehrdad Nahalparvari, Petros Karamanakos*, Tobias Geyer ${ }^{\dagger}$ \\ KTH Royal Institute of Technology \\ SE-10044 Stockholm, Sweden \\ *Tampere University \\ FI-33101 Tampere, Finland \\ ${ }^{\dagger}$ ABB Corporate Research \\ CH-5405 Baden-Dättwil, Switzerland \\ E-mail:mnah@kth.se,p.karamanakos@ieee.org,t.geyer@ieee.org
}

\section{Acknowledgments}

The first author would like to thank ABB Oy Drives, Helsinki, Finland, for the financial support.

\section{Keywords}

$\ll$ Control methods for electrical systems $\gg, \ll$ Optimal control $\gg, \ll$ Converter control $\gg, \ll$ Harmonics $\gg$, $\ll$ Modelling $\gg, \ll$ Pulse Width Modulation (PWM) $\gg, \ll$ Voltage Source Converter (VSC) $)$.

\begin{abstract}
This paper presents a direct model predictive control (MPC) scheme for a three-phase two-level gridconnected converter with an $L C L$ filter. Despite the absence of a modulator, a fixed switching frequency and a discrete current harmonic spectrum are achieved. With a refined objective function of the optimization problem underlying MPC, grid current distortions comparable to those of carrier-based pulse width modulation (CB-PWM) in idealized conditions are produced. Moreover, the grid current harmonics meet the stringent limits imposed by grid standards. A performance evaluation is conducted to highlight the potential benefits of the proposed method.
\end{abstract}

\section{Introduction}

Finite control set model predictive control (FCS-MPC) is a widely promoted MPC-based strategy in the field of power electronics. The underlying optimization problem-most often formulated as a reference tracking problem-is solved by enumerating the finite number of possible switch positions of the power electronic converter [1]. This implies that the converter switches are directly manipulated, thus the control and modulation problem are addressed in one computational stage [2]. Although direct control strategies provide fast transient responses, they operate the converter at a variable switching frequency, and, consequently, a non-discrete harmonic spectrum results [3]. This suggests that for a three-phase system significant harmonic components exist not only at non-triplen, odd integer multiples of the fundamental frequency, but also at even and inter-harmonics. This makes FCS-MPC unsuitable for the grid-side converter since the relevant grid codes—such as the IEEE 519 [4] and the IEC 61000-2-4 standard-impose tight limits on these harmonics.

To tackle the problem of variable switching frequency and spread harmonic spectra, several attempts have been made. A band-stop filter is combined with MPC in [5] which shapes the harmonic spectrum. Although this limits the range of the harmonics, it does not keep the switching frequency constant. In [6, 7], a deadbeat control approach with a modulator is proposed. However, a modulation stage deteriorates 


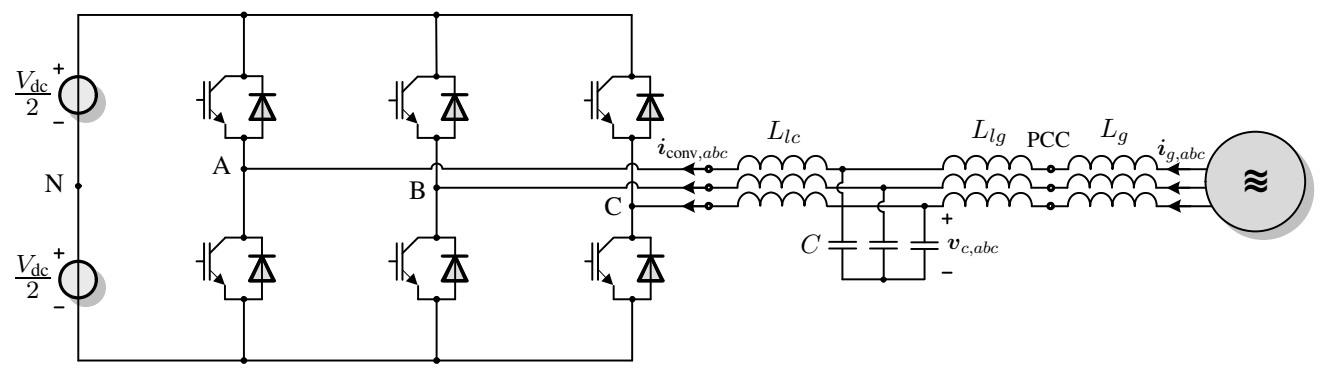

(a)

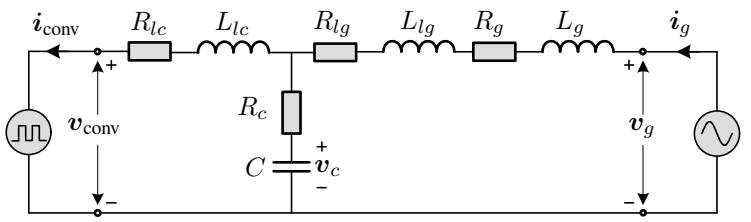

(b)

Fig. 1: (a) Two-level grid-connected converter with $L C L$ filter topology. (b) Equivalent circuit of the grid-connected converter with an $L C L$ filter in the stationary $(\alpha \beta)$ frame.

the fast transient responses inherent to direct MPC. Direct power control strategies are proposed in $[8,9]$, which result in a fixed switching frequency. However, because of the absence of any time constraints the implemented switching sequences are not symmetrical and, thus, non-discrete harmonic spectra result.

Other attempts involve FCS-MPC strategies that allow switching transitions to occur at any time instant within the sampling interval-rather than only at the discrete time steps-in an effort to reduce the ripple in the controlled variables. Consequently, the controller chooses the best possible time instant to apply a new switch position that will lead to an improved system performance. Nonetheless, these techniques either suffer from asymmetrical switching patterns and, thus, non-discrete harmonic spectra [10-13], or are prone to suboptimality [14].

In [15], a direct MPC scheme for a variable speed drive system is proposed which ensures a fixed switching frequency as well as a discrete harmonic spectrum, thus addressing the problems in [10-14]. To achieve this, each phase leg is constrained to switch once per sampling interval, i.e., a fixed modulation cycle is introduced akin to carrier-based pulse width modulation (CB-PWM). This paper adopts the proposed algorithm to control a three-phase two-level grid-connected converter equipped with an $L C L$ filter. Refinements, such as a linear approximation of the references and a longer prediction horizon, are proposed to improve the system performance. As it is shown, a discrete grid current harmonic spectrum is produced which abides by, e.g., the IEEE 519 grid standard [4]. Moreover, and in contrast to the common practice, an outer damping loop is not required despite the fact that the converter operates at a (fixed) switching frequency that may be less than twice the resonance frequency. Finally, fast transient responses inherent to direct MPC schemes are achieved during changes in the power references.

\section{Mathematical Model of the System}

The system under consideration consists of a three-phase two-level converter connected to the grid via an $L C L$ filter, see Fig. 1(a). In grid-connected applications, $L C L$ filters are preferred over $L$ filters because of their stronger attenuation of the harmonics beyond the resonance frequency. The dc-link voltage $V_{\mathrm{dc}}$ is assumed to be constant. MPC uses the system model to predict the future plant behavior and, subsequently, take necessary control actions. To this end, the discrete-time state-space model of the system in the stationary orthogonal reference frame ${ }^{1}(\alpha \beta)$ must be utilized.

The converter can be modeled as a gain. The two-level converter can produce at each phase terminal

\footnotetext{
${ }^{1}$ The operation $\boldsymbol{\xi}_{\alpha \beta}=\boldsymbol{K} \boldsymbol{\xi}_{a b c}$ is performed to map the variables $\boldsymbol{\xi}_{a b c}=\left[\begin{array}{lll}\xi_{a} & \xi_{b} & \xi_{c}\end{array}\right]^{T}$ in the three-phase (abc) system into the variables $\boldsymbol{\xi}_{\alpha \beta}=\left[\xi_{\alpha} \xi_{\beta}\right]^{T}$ in the stationary $(\alpha \beta)$ system.
} 
the two discrete voltage levels $-\frac{V_{\mathrm{dc}}}{2}$ and $\frac{V_{\mathrm{dc}}}{2}$, depending on the respective single-phase switch position $u_{x} \in \mathcal{U}=\{-1,1\}, x \in\{a, b, c\}$. Thus, the converter output voltage is given by [17] ${ }^{2}$

$$
\boldsymbol{v}_{\mathrm{conv}}=\frac{V_{\mathrm{dc}}}{2} \boldsymbol{K} \boldsymbol{u}_{a b c}
$$

where $\boldsymbol{K}$ is the amplitude-invariant Clarke transformation matrix and $\boldsymbol{u}_{a b c}=\left[u_{a} u_{b} u_{c}\right]^{T} \in \mathcal{U}^{3}$.

The dynamics of the system can be described by utilizing the equivalent circuit of the system illustrated in Fig. 1(b). Given that the dynamics of the converter current $\boldsymbol{i}_{\text {conv }}$, grid current $\boldsymbol{i}_{g}$, capacitor voltage $\boldsymbol{v}_{c}$, and grid voltage $\boldsymbol{v}_{g}$ fully describe the system in consideration, it follows that

$$
\begin{aligned}
\frac{\mathrm{d} \boldsymbol{i}_{\mathrm{conv}}}{\mathrm{d} t} & =\frac{1}{L_{l c}}\left(\boldsymbol{v}_{c}-\left(R_{l c}+R_{c}\right) \boldsymbol{i}_{\mathrm{conv}}+R_{c} \boldsymbol{i}_{g}-\boldsymbol{v}_{\mathrm{conv}}\right) \\
\frac{\mathrm{d} \boldsymbol{i}_{g}}{\mathrm{~d} t} & =\frac{1}{L_{l g}+L_{g}}\left(\boldsymbol{v}_{g}-\left(R_{l g}+R_{g}+R_{c}\right) \boldsymbol{i}_{g}+R_{c} \boldsymbol{i}_{\mathrm{conv}}-\boldsymbol{v}_{c}\right) \\
\frac{\mathrm{d} \boldsymbol{v}_{c}}{\mathrm{~d} t} & =\frac{1}{C}\left(\boldsymbol{i}_{g}-\boldsymbol{i}_{\mathrm{conv}}\right) \\
\frac{\mathrm{d} \boldsymbol{v}_{g}}{\mathrm{~d} t} & =\omega_{g}\left[\begin{array}{cc}
0 & -1 \\
1 & 0
\end{array}\right] \boldsymbol{v}_{g},
\end{aligned}
$$

where $R_{l c}\left(L_{l c}\right)$ is the converter-side filter resistance (inductance), $R_{l g}\left(L_{l g}\right)$ the grid-side filter resistance (inductance), and $R_{g}\left(L_{g}\right)$ the grid resistance (inductance). Moreover, $C$ and $R_{c}$ are the capacitance and internal resistance of the filter capacitor, respectively. Finally, $\omega_{g}$ is the angular grid frequency.

Using the converter model and the differential equations describing the system dynamics, the state-space model of the system can be derived. The continuous-time state-space model of the system is of the form

$$
\begin{aligned}
\frac{\mathrm{d} \boldsymbol{x}(t)}{\mathrm{d} t} & =\boldsymbol{F} \boldsymbol{x}(t)+\boldsymbol{G} \boldsymbol{K} \boldsymbol{u}_{a b c}(t) \\
\boldsymbol{y}(t) & =\boldsymbol{C} \boldsymbol{x}(t),
\end{aligned}
$$

where the state vector is chosen as $\boldsymbol{x}=\left[\boldsymbol{i}_{\text {conv }}^{T} \boldsymbol{i}_{g}^{T} \boldsymbol{v}_{c}^{T} \boldsymbol{v}_{g}^{T}\right]^{T} \in \mathbb{R}^{8}$. The output of the system is assumed to be the converter and grid current along with the capacitor voltage, i.e., $\boldsymbol{y}=\left[\boldsymbol{i}_{\text {conv }}^{T} \boldsymbol{i}_{g}^{T} \boldsymbol{v}_{c}^{T}\right]^{T} \in \mathbb{R}^{6}$. Considering the three-phase switch position $\boldsymbol{u}_{a b c}$ as the input to the system, the dynamics $\boldsymbol{F}$, input $\boldsymbol{G}$, and output $C$ matrices are defined as

$$
\begin{aligned}
& \boldsymbol{F}=\left[\begin{array}{cccc}
-\frac{R_{l c}+R_{c}}{L_{l c}} \boldsymbol{I}_{2} & \frac{R_{c}}{L_{l c}} \boldsymbol{I}_{2} & \frac{1}{L_{l c}} \boldsymbol{I}_{2} & \mathbf{0}_{2 \times 2} \\
\frac{R_{c}}{L_{l g}+L_{g}} \boldsymbol{I}_{2} & -\frac{R_{c}+R_{l g}+R_{g}}{L_{l g}+L_{g}} \boldsymbol{I}_{2} & -\frac{1}{L_{l g}+L_{g}} \boldsymbol{I}_{2} & \frac{1}{L_{l g}+L_{g}} \boldsymbol{I}_{2} \\
-\frac{1}{C} \boldsymbol{I}_{2} & \frac{1}{C} \boldsymbol{I}_{2} & \mathbf{0}_{2 \times 2} & \mathbf{0}_{2 \times 2} \\
\mathbf{0}_{2 \times 2} & \mathbf{0}_{2 \times 2} & \mathbf{0}_{2 \times 2} & \omega_{g}\left[\begin{array}{cc}
0 & -1 \\
1 & 0
\end{array}\right]
\end{array}\right] \\
& \boldsymbol{G}=-\frac{V_{\mathrm{dc}}}{2 L_{l c}}\left[\begin{array}{ll}
\boldsymbol{I}_{2} & \mathbf{0}_{2 \times 6}
\end{array}\right]^{T}, \text { and } \boldsymbol{C}=\left[\begin{array}{ll}
\boldsymbol{I}_{6} & \mathbf{0}_{6 \times 2}
\end{array}\right],
\end{aligned}
$$

where $\boldsymbol{I}$ and $\mathbf{0}$ are identity and zero matrices of appropriate dimensions, respectively.

Finally, the discrete-time state-space model of the system can be derived based on exact discretization as

$$
\begin{aligned}
\boldsymbol{x}(k+1) & =\boldsymbol{A} \boldsymbol{x}(k)+\boldsymbol{B} \boldsymbol{K} \boldsymbol{u}_{a b c}(k) \\
\boldsymbol{y}(k) & =\boldsymbol{C} \boldsymbol{x}(k),
\end{aligned}
$$

\footnotetext{
${ }^{2}$ Throughout the text, any quantity in the $\alpha \beta$ plane bears no subscript whereas variables in the $a b c$ plane are denoted appropriately.
} 


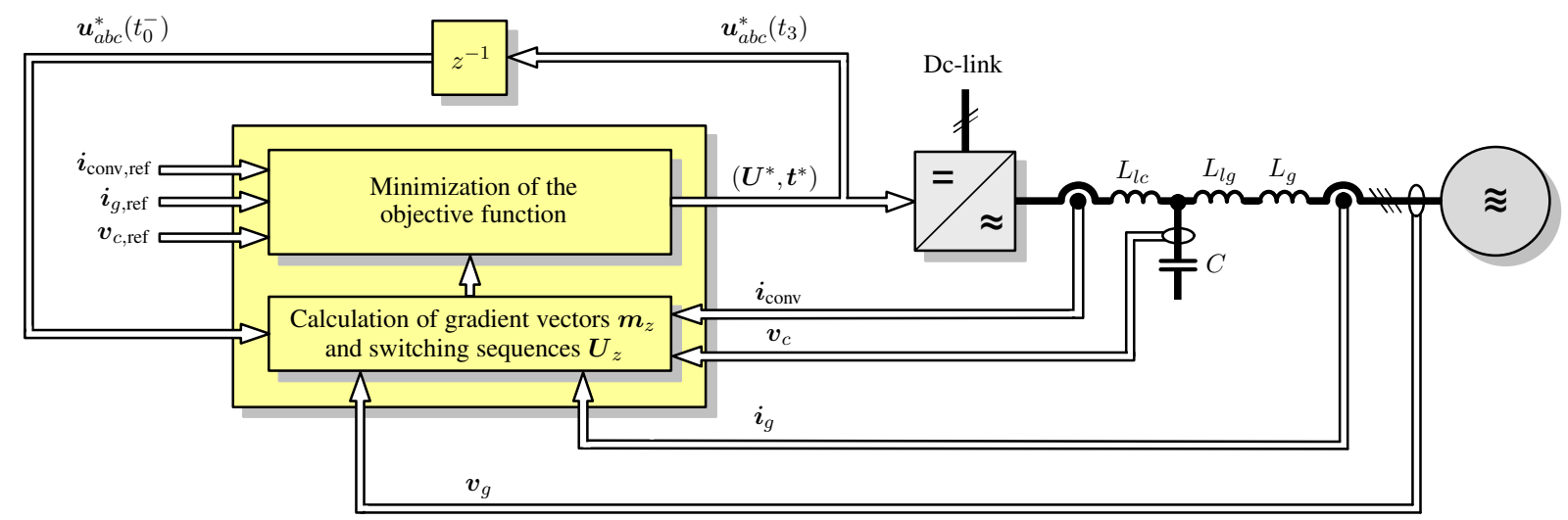

Fig. 2: Block diagram of the control scheme for a two-level grid-connected converter with an $L C L$ filter. with $\boldsymbol{A}=\mathrm{e}^{\boldsymbol{F} T_{s}}$ and $\boldsymbol{B}=-\boldsymbol{F}^{-1}\left(\boldsymbol{I}_{8}-\boldsymbol{A}\right) \boldsymbol{G}$, where $\mathbf{e}$ is the matrix exponential, $T_{s}$ the sampling interval, and $k \in \mathbb{N}$.

\section{Direct Model Predictive Control with Fixed Switching Frequency}

The block diagram of the proposed direct MPC algorithm is depicted in Fig. 2. Since the discussed scheme is a direct control strategy the converter switches are directly manipulated, i.e., a dedicated modulation stage is not required. Despite the absence of a modulator, though, a constant switching frequency is achieved, as explained in the sequel of this section.

\section{Control Problem}

The objective of the proposed control strategy is to minimize the ripple of the controlled variables $y$ while guaranteeing a fixed switching frequency. The latter is realized by forcing each phase to switch once per sampling interval $T_{s}$. To explain this in more detail, let the entries of $\boldsymbol{t}=\left[\begin{array}{lll}t_{1} & t_{2} & t_{3}\end{array}\right]^{T}$, with $0<t_{1}<t_{2}<t_{3}<T_{s}$, be the switching time instants; at these time instants, a new switch position $\boldsymbol{u}_{a b c}\left(t_{z}\right), z \in\{1,2,3\}$, is applied to the converter. At the beginning of the current sampling interval $\left(t_{0} \equiv 0\right)$, the switch position $\boldsymbol{u}_{a b c}\left(t_{0}\right)$, which was applied at the end of the previous sampling interval, is applied to the converter, i.e., $\boldsymbol{u}_{a b c}\left(t_{0}\right)=\boldsymbol{u}_{a b c}\left(t_{0}^{-}\right)$. At time instant $t_{1}$, a new switch position $\boldsymbol{u}_{a b c}\left(t_{1}\right)$ is applied to the converter that leads to a switching in one of the three phases. Following, at $t_{2}$, one of the two - thus far-inactive phases switches by applying $\boldsymbol{u}_{a b c}\left(t_{2}\right)$. Finally, the last phase switches at time instant $t_{3}$, when the switch position $\boldsymbol{u}_{a b c}\left(t_{3}\right)$ is applied. Therefore, in each sampling interval the switching sequence $\boldsymbol{U}=\left[\boldsymbol{u}_{a b c}^{T}\left(t_{0}\right) \boldsymbol{u}_{a b c}^{T}\left(t_{1}\right) \boldsymbol{u}_{a b c}^{T}\left(t_{2}\right) \boldsymbol{u}_{a b c}^{T}\left(t_{3}\right)\right]^{T}$ is implemented. The following example is provided for better understanding.

Example: Consider the two-level converter in Fig. 1(a). Assume that in all three phase legs the lower switch is on at $t \equiv t_{0}^{-}$. Thus, it follows that at $t_{0}$ the switch position $\boldsymbol{u}_{a b c}\left(t_{0}\right)=[-1-1-1]^{T}$ is applied to the converter. As illustrated in Fig. 3(a), the three-phase switch positions $\left[\begin{array}{lll}-1 & 1-1\end{array}\right]^{T},\left[\begin{array}{lll}-1 & 1 & 1\end{array}\right]^{T}$ and $\left[\begin{array}{lll}1 & 1 & 1\end{array}\right]^{T}$ are applied at time instants $t_{1}, t_{2}$ and $t_{3}$, respectively, with $0<t_{1}<t_{2}<t_{3}<T_{s}$. As it is hinted, six possible switching sequences $\boldsymbol{U}$ exist, since the three phases can switch in six different chronological orders, e.g., phase $b$ can switch first, followed by phases $c$ and $a$, or assuming phase $b$ switches first, then phase $a$ switches second and phase $c$ third, etc.

\section{Control Method}

To meet the first control objective, i.e., the minimization of the ripple of the output variables $\boldsymbol{y}$, the deviation of these variables from their reference values $\boldsymbol{y}_{\text {ref }}$ is captured with an objective function. To this end, the weighted squared rms error of the output variables can be penalized. However, to avoid ending up with a computationally intractable control problem, we opt for the weighted sampled squared error at the switching instants $t_{z}$, and the end of the sampling interval, i.e., $t=T_{s}$. By doing so, the problem remains computationally feasible while the rms error is approximated in a coarse, yet effective, way [15]. 


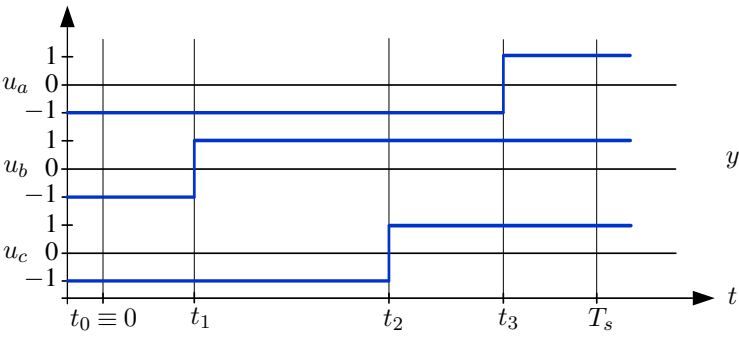

(a) Three-phase switch position

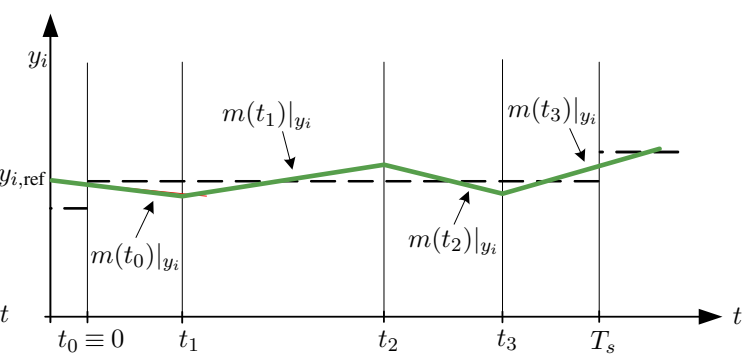

(b) Controlled variable $y_{i}$

Fig. 3: (a) Switching sequence $b \rightarrow c \rightarrow a$ assuming $\boldsymbol{u}_{a b c}\left(t_{0}\right)=\boldsymbol{u}_{a b c}\left(t_{0}^{-}\right)=[-1-1-1]^{T}$. (b) Example evolution of an (arbitrary) output variable within one sampling interval.

Table I: Possible switching sequences for the single-phase switching transitions for a two-step horizon

\begin{tabular}{|cccccc|}
\hline \multicolumn{3}{|c|}{$1^{\text {st }}$} & sampling interval & \multicolumn{3}{c|}{$2^{\text {nd }}$ sampling interval } \\
\hline First & Second & Third & First & Second & Third \\
\hline$a$ & $b$ & $c$ & $c$ & $b$ & $a$ \\
$a$ & $c$ & $b$ & $b$ & $c$ & $a$ \\
$b$ & $a$ & $c$ & $c$ & $a$ & $b$ \\
$b$ & $c$ & $a$ & $a$ & $c$ & $b$ \\
$c$ & $a$ & $b$ & $b$ & $a$ & $c$ \\
$c$ & $b$ & $a$ & $a$ & $b$ & $c$ \\
\hline
\end{tabular}

Furthermore, a two-step horizon is employed to enable the controller to make better educated decisions about the future evolution of the controlled system. As shown, e.g., in [16], long horizons have a beneficial effect on the system performance, especially when higher-order multiple-input multiple-output (MIMO) systems, as the one in this work, are considered. To this end, the switching sequence in the first (kth) sampling interval is defined as $\boldsymbol{U}(k)=\left[\boldsymbol{u}_{a b c}^{T}\left(t_{0}(k)\right) \boldsymbol{u}_{a b c}^{T}\left(t_{1}(k)\right) \boldsymbol{u}_{a b c}^{T}\left(t_{2}(k)\right) \boldsymbol{u}_{a b c}^{T}\left(t_{3}(k)\right)\right]^{T}$ and the corresponding vector of switching instants is $\boldsymbol{t}(k)=\left[t_{1}(k) t_{2}(k) t_{3}(k)\right]^{T}$. Thereby, with a prediction horizon over two sampling intervals, the applied switching sequence and the corresponding vector of switching instants are defined as

$$
\begin{aligned}
\boldsymbol{U} & =\left[\boldsymbol{U}^{T}(k) \boldsymbol{U}^{T}(k+1)\right]^{T} \\
\boldsymbol{t} & =\left[\boldsymbol{t}^{T}(k) \boldsymbol{t}^{T}(k+1)\right]^{T} .
\end{aligned}
$$

By increasing the prediction horizon to two sampling intervals, however, a subsequent increase in the computational complexity results. Specifically, $6^{2}=36$ possible switching sequences $\boldsymbol{U}$ with the corresponding switching instants $t$ need to be considered. To keep the computational complexity at bay, we define $\boldsymbol{U}(k+1)=\left[\boldsymbol{u}_{a b c}^{T}\left(t_{3}(k)\right) \boldsymbol{u}_{a b c}^{T}\left(t_{2}(k)\right) \boldsymbol{u}_{a b c}^{T}\left(t_{1}(k)\right) \boldsymbol{u}_{a b c}^{T}\left(t_{0}(k)\right)\right]^{T}$, i.e., the switching sequence in the second sampling interval $\boldsymbol{U}(k+1)$ mirrors (with respect to $T_{s}$ ) that in the first interval $\boldsymbol{U}(k)$, akin, e.g., to the switching pattern in space vector modulation (SVM). As a result, only six unique switching sequences need to be evaluated. Table I shows the possible sequences for the single-phase switching transitions over a two-step horizon. Given the above, the proposed objective function is

$$
\begin{aligned}
J= & \sum_{\ell=k}^{k+1}\left\|\boldsymbol{y}_{\mathrm{ref}}\left(t_{1}(\ell)\right)-\boldsymbol{y}\left(t_{1}(\ell)\right)\right\|_{\boldsymbol{Q}}^{2}+\left\|\boldsymbol{y}_{\mathrm{ref}}\left(t_{2}(\ell)\right)-\boldsymbol{y}\left(t_{2}(\ell)\right)\right\|_{\boldsymbol{Q}}^{2}+\left\|\boldsymbol{y}_{\mathrm{ref}}\left(t_{3}(\ell)\right)-\boldsymbol{y}\left(t_{3}(\ell)\right)\right\|_{\boldsymbol{Q}}^{2} \\
& +\left\|\boldsymbol{y}_{\mathrm{ref}}\left(T_{s}(\ell)\right)-\boldsymbol{y}\left(T_{s}(\ell)\right)\right\|_{\boldsymbol{Q}}^{2},
\end{aligned}
$$

where $Q \in \mathbb{R}^{6 \times 6}$ is a diagonal positive definite weighting matrix, the entries of which prioritize the tracking accuracy among the different controlled variables. Note that in (7), the reference values within the first and second sampling intervals are approximated by linearly interpolating between the references at time steps $k$ and $k+1$, and $k+1$ and $k+2$, respectively as seen in Fig. 4. An alternative would 


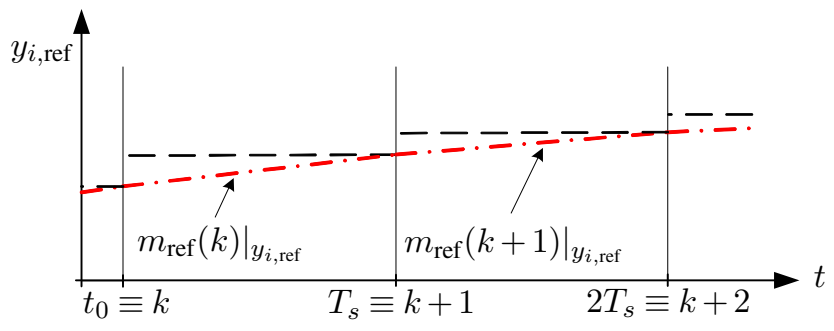

Fig. 4: Linear approximation of the reference values of an (arbitrary) output variable $y_{i}$,ref over two sampling intervals (dash-dotted red line). The sampled (constant) reference is shown with a dashed black line.

be to use a sample and hold approach, i.e., to assume constant references at each sampling interval, but this would result in a worse tracking performance and is thus avoided. When linearly approximating the references, the gradients (slopes) are given by

$$
\boldsymbol{m}_{\mathrm{ref}}(\ell)=\frac{\boldsymbol{y}_{\mathrm{ref}}(\ell+1)-\boldsymbol{y}_{\mathrm{ref}}(\ell)}{T_{s}},
$$

with $\ell=k, k+1$.

In addition, as shown in [15], the controlled variables can be assumed to evolve linearly with constant gradients $\boldsymbol{m}(t)=\mathrm{d} \boldsymbol{y}(t) / \mathrm{d} t \in \mathbb{R}^{6}$ within one sampling interval $T_{s}$. Such an assumption is valid, considering that $T_{s} \ll T_{1}$, where $T_{1}$ is the fundamental period. For the same reason, the vector of gradients $\boldsymbol{m}$ can be considered constant throughout the sampling interval. This also implies that all four gradients within the sampling interval depend on the state at $t_{0}$, i.e., $\boldsymbol{x}\left(t_{0}\right)$, rather than the state at the intermediate switching time instants. Thus, the evolution of the output variables within the four subintervals $\left[0, t_{1}\right)$, $\left[t_{1}, t_{2}\right),\left[t_{2}, t_{3}\right)$ and $\left[t_{3}, T_{s}\right)$ is described by

$$
\boldsymbol{m}\left(t_{i}\right)=\frac{\mathrm{d} \boldsymbol{y}\left(t_{i}\right)}{\mathrm{d} t}=\boldsymbol{C} \frac{\mathrm{d} \boldsymbol{x}\left(t_{i}\right)}{\mathrm{d} t}=\boldsymbol{C}\left(\boldsymbol{F} \boldsymbol{x}\left(t_{0}\right)+\boldsymbol{G} \boldsymbol{K} \boldsymbol{u}_{a b c}\left(t_{i}\right)\right),
$$

with $i \in\{0,1,2,3\}$, see Fig. 3(b). With (9), the output variables at $t_{i}, i \in\{1,2,3,4\}$, can be given by

$$
\boldsymbol{y}\left(t_{i}(\ell)\right)=\boldsymbol{y}\left(t_{i-1}(\ell)\right)+\boldsymbol{m}\left(t_{i-1}(\ell)\right)\left(t_{i}(\ell)-t_{i-1}(\ell)\right),
$$

where $t_{4}=T_{s}$.

Utilizing (10), and by means of algebraic manipulations, the objective function (7) can be rewritten in the vector form as

$$
J=\|\boldsymbol{r}-\boldsymbol{M t}\|_{\widetilde{\boldsymbol{Q}}}^{2}
$$

where the optimization variable $t \in \mathbb{R}^{6}$ is given by (6b). Furthermore, $r \in \mathbb{R}^{48}$ and $M \in \mathbb{R}^{48 \times 6}$ are time-invariant and given in the Appendix. Finally, $\widetilde{\boldsymbol{Q}}=\operatorname{diag}(\boldsymbol{Q}, \ldots, \boldsymbol{Q})$.

\section{Control Algorithm}

The pseudocode of the scheme is summarized in Algorithm 1.

Before solving the optimization problem formulated in the framework of MPC, two steps have to be taken. First, the possible gradients $\boldsymbol{m}_{z}\left(t_{0}\right)$ are computed with (9) based on the measured state at $t_{0}$, i.e., $\boldsymbol{x}\left(t_{0}\right)$, and the possible three-phase switch positions $\boldsymbol{u}_{a b c}$. In doing so, seven unique output vector gradients $\boldsymbol{m}_{z}, z \in\{0,1, \ldots, 6\}$, are computed. Second, the controller enumerates the six possible switching sequences within the two sampling intervals as shown in Table I. 


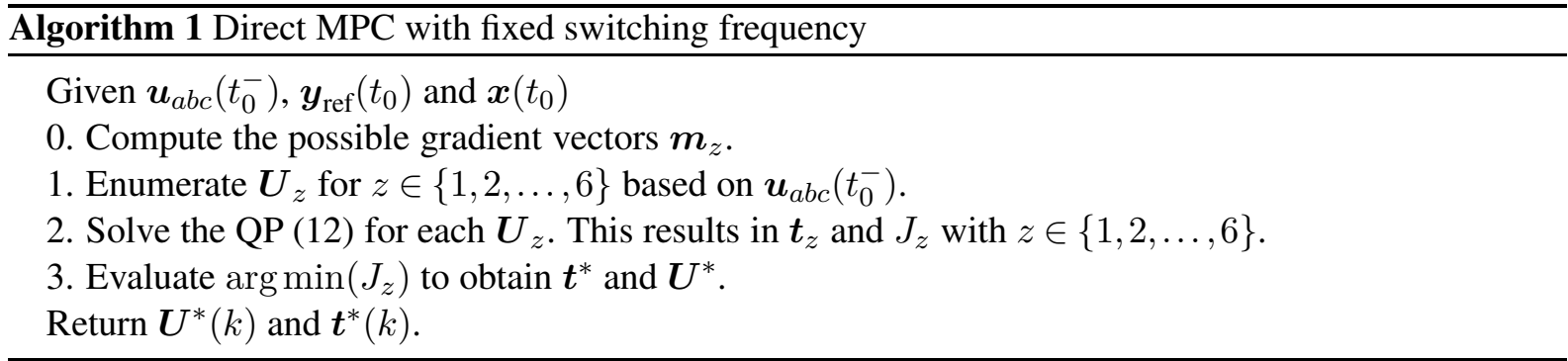

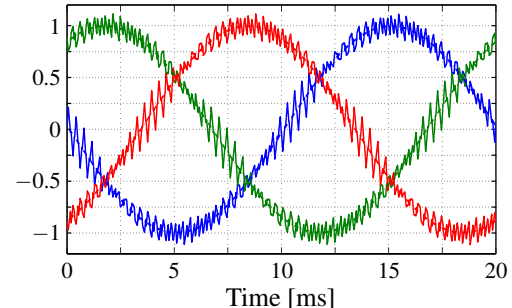

(a) Three-phase convert

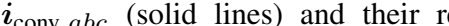
ences (dash-dotted lines).

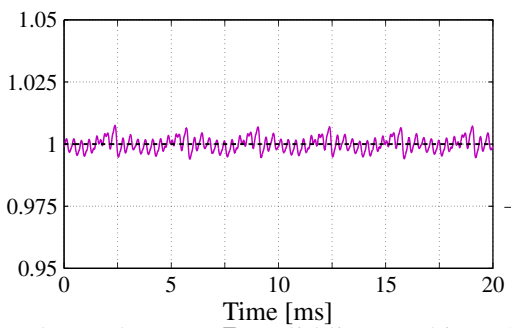

(d) Real power $P$ (solid line) and its reference (dash-dotted line).

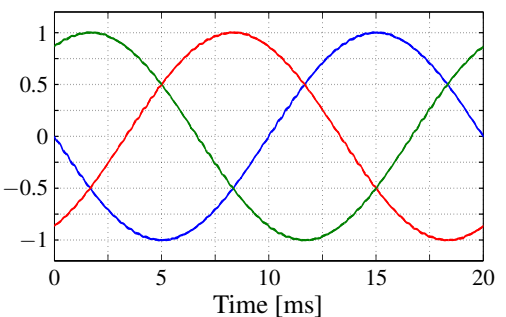

(b) Three-phase grid currents $\boldsymbol{i}_{g, a b c}$ (solid lines) and their references (dash-
dotted lines).

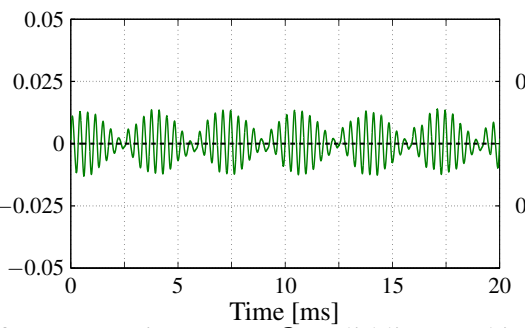

(e) Reactive power $Q$ (solid reference (dash-dotted line).

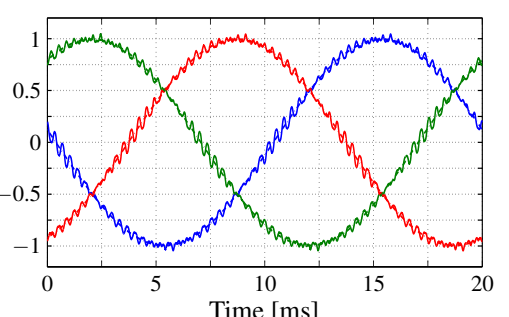

(c) Three-phase capacitor voltages $\boldsymbol{v}_{c, a b c}$ (solid lines) and their references (dash-dotted lines).

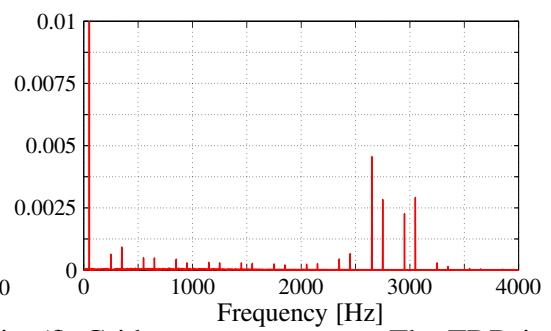

(f) Grid current spectrum. The TDD is $0.69 \%$.

Fig. 5: Simulated waveforms produced by direct model predictive control at steady-state operation for unity power factor. The switching frequency is $2850 \mathrm{~Hz}$.

Following, for each candidate switching sequence, the optimization problem

$$
\begin{array}{ll}
\underset{\boldsymbol{t} \in \mathbb{R}^{6}}{\operatorname{minimize}} & J=\|\boldsymbol{r}-\boldsymbol{M t}\|_{\widetilde{\boldsymbol{Q}}}^{2} \\
\text { subject to } & 0<t_{1}(k)<t_{2}(k)<t_{3}(k)<T_{s}<t_{1}(k+1)<t_{2}(k+1)<t_{3}(k+1)<2 T_{s},
\end{array}
$$

is solved. Problem (12) is a convex quadratic program (QP), which can be efficiently solved using offthe-shelf solvers. By solving the QP for each one of the six switching sequences $\boldsymbol{U}_{z}, z \in\{1,2, \ldots, 6\}$, six triplets of switching instants $\boldsymbol{t}_{z}$, switching sequences $\boldsymbol{U}_{z}$ and values of the objective function $J_{z}$ result. The triplet with the minimum associated cost, i.e., the one with the minimum value of $J_{z}$, is the optimal one. Therefore, the optimal switching sequence $U^{*}$ and the corresponding vector of switching time instants $\boldsymbol{t}^{*}$ are obtained. However, according to the receding horizon policy [18], only the elements corresponding to the $k$ th sampling interval, i.e., $\boldsymbol{U}^{*}(k)$ and $\boldsymbol{t}^{*}(k)$, are applied to the converter at time step $k$, whereas the rest elements are discarded. The procedure is repeated at the next time step $k+1$ based on new measurements over a prediction horizon that is shifted by one sampling interval.

\section{Performance Evaluation}

In this section, simulation results are provided to verify the performance of the control method on a two-level grid-connected converter with an $L C L$ filter. An idealized setup is assumed, i.e., second-order effects, such as measurement noise, deadtimes, etc., are neglected. The grid has a rated voltage of $400 \mathrm{~V}$, short-circuit ratio 20 , the impedance ratio $X / R=7$ and a rated frequency of $50 \mathrm{~Hz}$. The nominal power of the converter is $12.5 \mathrm{kVA}$ and its rated current is $18 \mathrm{~A}$. Furthermore, the dc-link voltage is $650 \mathrm{~V}$ and the filter parameters are $L_{l c}=3.3 \mathrm{mH}, L_{l g}=3 \mathrm{mH}, C=8.8 \mu \mathrm{F}, R_{l c}=100 \mathrm{~m} \Omega, R_{l g}=70 \mathrm{~m} \Omega$ and 


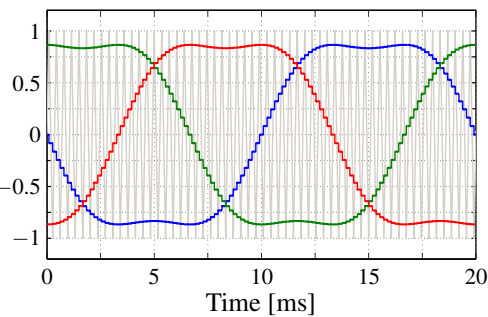

(a) Three-phase modulating signal and (b) triangular carrier.

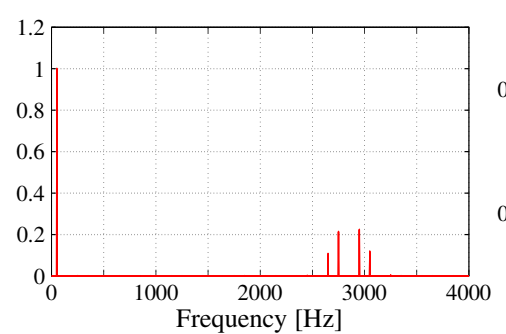

(b) Harmonic spectrum of differentialmode converter voltage.

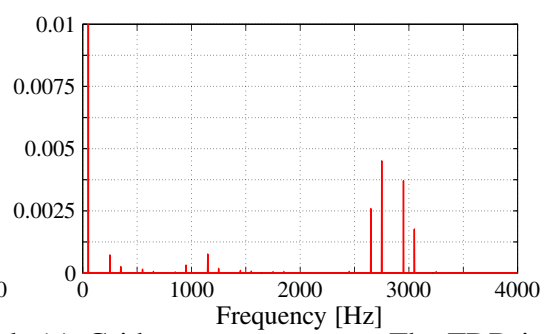

(c) Grid current spectrum. The TDD is $0.68 \%$.

Fig. 6: Simulated waveforms produced by open-loop asymmetric regularly sampled CB-PWM with third harmonic injection.

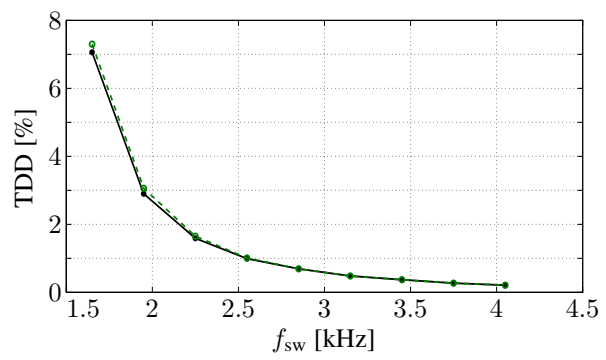

Fig. 7: Grid current TDD produced by MPC (green) and CB-PWM (black) for a range of switching frequencies.

$R_{c}=0.8 \mathrm{~m} \Omega$. Thus, the dominant resonance frequency of the filter is at $1203.3 \mathrm{~Hz}$. The sampling interval is set to $175.43 \mu$ s such that a constant switching frequency of $f_{\mathrm{sw}}=2850 \mathrm{~Hz}$ results. The entries of the weighting matrix $\boldsymbol{Q}$ are selected by trial and error as $\boldsymbol{Q}=\operatorname{diag}(1,1,9,9,0.9,0.9)$, with tracking of the grid current prioritized. All results are shown in the per unit (p.u.) system.

The steady-state performance of the system is shown in Fig. 5 for one fundamental period. Steadystate operation at unity power factor (pf), i.e., $P=1$ p.u. and $Q=0$ p.u., is considered. As can be seen in Figs. 5(a)-5(c), the controlled variables accurately track their reference values while operation under unity power factor is achieved (see Figs. 5(d) and 5(e)). Fig. 5(f) shows the harmonic spectrum of the grid current. The harmonics are at odd, non-triplen multiples of the fundamental frequency. The total demand distortion (TDD) which quantifies the quality of the grid current waveform is $0.69 \%$. Moreover, as can be seen in Fig. 5(f), the constraints on the harmonic amplitudes of the grid currents at the PCC, imposed by the IEEE 519 standard [4], are met. More importantly, although the ratio between the switching and resonance frequency is $\approx 2.37$, the resonance frequency is not excited despite the absence of an additional active damping loop. Thus, as it is implied, the proposed method can operate the converter at relatively low switching frequencies, thus reducing the switching power losses.

As a benchmark, asymmetric regularly sampled CB-PWM with third harmonic injection is implemented for operation at the same switching frequency, i.e., $f_{\mathrm{sw}}=2850 \mathrm{~Hz}$. This can be interpreted as having a simple closed-loop linear controller with a very low bandwidth. As before, an idealized setup without disturbances, delays, and model imperfections is assumed. Fig. 6(a) illustrates the three-phase modulating signal and the triangular carrier waveform, while Figs. 6(b) and 6(c) show the spectrum of converter (differential-mode) output voltage and grid current, respectively. As can be seen in Fig. 6(c), the harmonics produced by CB-PWM are of the same order, but slightly different amplitude, compared with those produced by direct MPC. Despite these small differences, the grid current TDD is $0.68 \%$, i.e., very similar to the one obtained by the proposed method. This similarity is also verified in Fig. 7, which depicts the TDD of the grid current obtained by the proposed MPC scheme and open-loop CB-PWM for a range of switching frequencies between $1650 \mathrm{~Hz}$ and $4050 \mathrm{~Hz}$. As can be seen, both methods perform alike in terms of current distortions, even at switching frequencies closer to the filter resonance frequency, and in spite of the lack of an active damping loop in the presented control scheme.

The performance of the proposed direct MPC algorithm is investigated during step changes in the power 


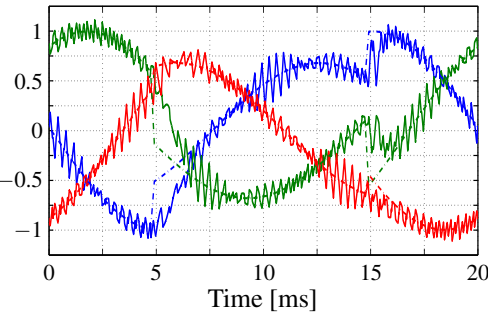

(a) Three-phase converter $\boldsymbol{i}_{\text {conv, } a b c}$ (solid lines) and their refer- (solid lines) and their references (dashences (dash-dotted lines).

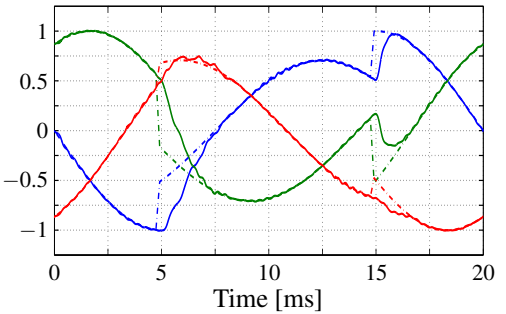

dotted lines).

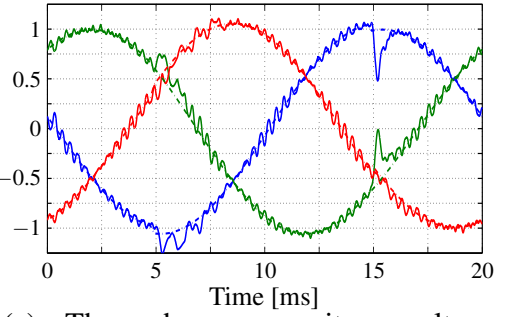

(c) Three-phase capacitor voltages $\boldsymbol{v}_{c, a b c}$ (solid lines) and their references (dash-dotted lines).

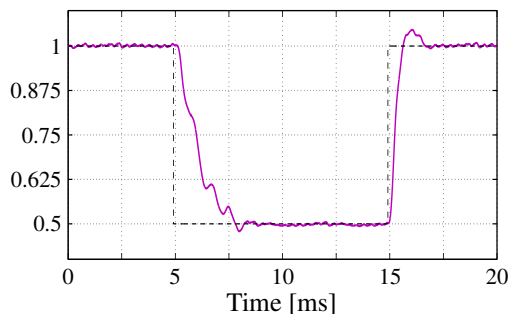

(d) Real power $P$ (solid line) and its reference (dash-dotted line).

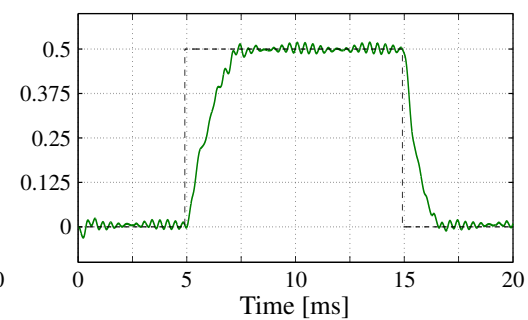

(e) Reactive power $Q$ (solid line) and its reference (dash-dotted line).

Fig. 8: Power reference steps for direct MPC. The switching frequency is $2850 \mathrm{~Hz}$.

reference, see Fig. 8. While operating at nominal operating conditions $(\mathrm{pf}=1)$, real and reactive power reference steps of 0.5 p.u. are imposed at instant $t \approx 5 \mathrm{~ms}$; the references are changed back at $t \approx 15 \mathrm{~ms}$ so that nominal operation is regained. The controlled variables-and consequently the powers-follow their references accurately. MPC, being a direct controller, makes decisions such that the error in the controlled variables is eliminated as quickly as possible. Effectively, the settling time is affected by the available voltage margin. For example, the slower dynamics during the step-down-compared to the step-up - change in the real power reference are due to the smaller voltage margin available when operating in nominal grid condition.

\section{Conclusions}

This paper presents a direct MPC with fixed switching frequency scheme for a three-phase two-level grid-connected converter with an $L C L$ filter. Owing to the inherent characteristics of the method as well as the proposed refinements, the grid current spectrum meets the relevant grid codes, while an additional active damping loop is not required. Furthermore, thanks to its MIMO approach, all controlled variables are controlled such that an optimal trade-off—adjusted by the penalty matrix $Q$-between their coupling and deviations from their references is achieved. Finally, the fast transient responses that characterize direct control schemes, as the proposed one, are still present. Future work may include experimental verification of the proposed control scheme and its effectiveness under unbalanced grid conditions.

\section{Appendix}

The time-invariant vector $\boldsymbol{r}$ and matrix $\boldsymbol{M}$ in (11) are given by

$$
\boldsymbol{r}=\left[\begin{array}{c}
\boldsymbol{y}_{\text {ref }}\left(t_{0}\right)-\boldsymbol{y}\left(t_{0}\right) \\
\boldsymbol{y}_{\text {ref }}\left(t_{0}\right)-\boldsymbol{y}\left(t_{0}\right) \\
\boldsymbol{y}_{\text {ref }}\left(t_{0}\right)-\boldsymbol{y}\left(t_{0}\right) \\
\boldsymbol{y}_{\mathrm{ref}}\left(T_{s}\right)-\boldsymbol{y}\left(t_{0}\right)-\boldsymbol{m}\left(t_{3}(k)\right) T_{s} \\
\boldsymbol{y}_{\text {ref }}\left(T_{s}\right)-\boldsymbol{y}\left(t_{0}\right) \\
\boldsymbol{y}_{\text {ref }}\left(T_{s}\right)-\boldsymbol{y}\left(t_{0}\right) \\
\boldsymbol{y}_{\mathrm{ref}}\left(T_{s}\right)-\boldsymbol{y}\left(t_{0}\right) \\
\boldsymbol{y}_{\mathrm{ref}}\left(2 T_{s}\right)-\boldsymbol{y}\left(t_{0}\right)-\boldsymbol{m}\left(t_{3}(k+1)\right) 2 T_{s}
\end{array}\right],\left[\begin{array}{cccccc}
\boldsymbol{m}_{t_{0}} & \mathbf{0}_{6} & \mathbf{0}_{6} & \mathbf{0}_{6} & \mathbf{0}_{6} & \mathbf{0}_{6} \\
\boldsymbol{m}_{0} & \boldsymbol{m}_{t_{1}} & \mathbf{0}_{6} & \mathbf{0}_{6} & \mathbf{0}_{6} & \mathbf{0}_{6} \\
\boldsymbol{m}_{0} & \boldsymbol{m}_{1} & \boldsymbol{m}_{t_{2}} & \mathbf{0}_{6} & \mathbf{0}_{6} & \mathbf{0}_{6} \\
\boldsymbol{m}_{0} & \boldsymbol{m}_{1} & \boldsymbol{m}_{2} & \mathbf{0}_{6} & \mathbf{0}_{6} & \mathbf{0}_{6} \\
\boldsymbol{m}_{0} & \boldsymbol{m}_{1} & \boldsymbol{m}_{2} & \widetilde{\boldsymbol{m}}_{t_{0}} & \mathbf{0}_{6} & \mathbf{0}_{6} \\
\boldsymbol{m}_{0} & \boldsymbol{m}_{1} & \boldsymbol{m}_{2} & \widetilde{\boldsymbol{m}}_{0} & \widetilde{\boldsymbol{m}}_{t_{1}} & \mathbf{0}_{6} \\
\boldsymbol{m}_{0} & \boldsymbol{m}_{1} & \boldsymbol{m}_{2} & \widetilde{\boldsymbol{m}}_{0} & \widetilde{\boldsymbol{m}}_{1} & \widetilde{\boldsymbol{m}}_{t_{2}} \\
\boldsymbol{m}_{0} & \boldsymbol{m}_{1} & \boldsymbol{m}_{2} & \widetilde{\boldsymbol{m}}_{0} & \widetilde{\boldsymbol{m}}_{1} & \widetilde{\boldsymbol{m}}_{2}
\end{array}\right],
$$


with

$$
\begin{aligned}
& \boldsymbol{m}_{t_{i}}=\boldsymbol{m}\left(t_{i}(k)\right)-\boldsymbol{m}_{\mathrm{ref}}(k), \\
& \widetilde{\boldsymbol{m}}_{t_{i}}=\boldsymbol{m}\left(t_{i}(k+1)\right)-\boldsymbol{m}_{\mathrm{ref}}(k+1),
\end{aligned}
$$

$$
\begin{aligned}
\boldsymbol{m}_{i} & =\boldsymbol{m}\left(t_{i}(k)\right)-\boldsymbol{m}_{t_{i+1}}(k), \\
\widetilde{\boldsymbol{m}}_{i} & =\boldsymbol{m}\left(t_{i}(k+1)\right)-\boldsymbol{m}_{t_{i+1}}(k+1),
\end{aligned}
$$

where $i \in\{0,1,2\}$, and $t_{0}(k+1)=T_{s}$.

\section{References}

[1] P. Cortés, M. P. Kazmierkowski, R. M. Kennel, D. E. Quevedo, and J. Rodríguez, "Predictive control in power electronics and drives," in IEEE Trans. Ind. Electron., vol. 55, no. 12, pp. 4312-4324, Dec. 2008.

[2] T. Geyer, Model predictive control of high power converters and industrial drives. Hoboken, NJ: Wiley, 2016.

[3] — - "A comparison of control and modulation schemes for medium-voltage drives: Emerging predictive control concepts versus PWM-based schemes," in IEEE Trans. Ind. Appl., vol. 47, no. 3, pp. 1380-1389, May-Jun. 2011.

[4] IEEE Std 519-2014 (Revision of IEEE Std 519-1992), "IEEE recommended practices and requirements for harmonic control in electrical power systems," Jun. 2014, pp. 1-29.

[5] P. Cortés, J. Rodríguez, D. E. Quevedo, and C. Silva, "Predictive current control strategy with imposed load current spectrum," in IEEE Trans. Power Electron., vol. 23, no. 2, pp. 612-618, Mar. 2008.

[6] A. Bouafia, J. Gaubert, and F. Krim, "Predictive direct power control of three-phase pulsewidth modulation (PWM) rectifier using space-vector modulation (SVM)," in IEEE Trans. Power Electron., vol. 25, no. 1, pp. 228-236, Jan. 2010.

[7] R. O. Ramírez, J. R. Espinoza, F. Villarroel, E. Maurelia, and M. E. Reyes, "A novel hybrid finite control set model predictive control scheme with reduced switching," in IEEE Trans. Ind. Electron., vol. 61, no. 11, pp. 5912-5920, Nov. 2014.

[8] S. Vazquez, A. Marquez, R. Aguilera, D. Quevedo, J. I. Leon, and L. G. Franquelo, "Predictive optimal switching sequence direct power control for grid-connected power converters," in IEEE Trans. Ind. Electron., vol. 62, no. 4, pp. 2010-2020, Apr. 2015.

[9] S. A. Larrinaga, M. A. R. Vidal, E. Oyarbide, and J. R. T. Apraiz, "Predictive control strategy for dc/ac converters based on direct power control," in IEEE Trans. Ind. Electron., vol. 54, no. 3, pp. 1261-1271, Jun. 2007.

[10] P. Karamanakos, P. Stolze, R. M. Kennel, S. Manias, and H. du T. Mouton, "Variable switching point predictive torque control of induction machines," IEEE J. Emerg. Sel. Topics Power Electron., vol. 2, no. 2, pp. 285-295, Jun. 2014.

[11] P. Karamanakos, A. Ayad, and R. Kennel, "A variable switching point predictive current control strategy for quasi-z-source inverters,” IEEE Trans. Ind. Appl., vol. 54, no. 2, pp. 1469-1480, Mar. 2018.

[12] L. Tarisciotti, P. Zanchetta, A. Watson, S. Bifaretti, and J. C. Clare, "Modulated model predictive control for a seven-level cascaded h-bridge back-to-back converter," IEEE Trans. Ind. Electron., vol. 61, no. 10, pp. 5375-5383, Oct. 2014.

[13] M. Tomlinson, H. d. T. Mouton, R. Kennel, and P. Stolze, "A fixed switching frequency scheme for finitecontrol-set model predictive control-Concept and algorithm," IEEE Trans. Ind. Electron., vol. 63, no. 12, pp. 7662-7670, Dec. 2016.

[14] Y. Zhang, W. Xie, Z. Li, and Y. Zhang, "Low-complexity model predictive power control: Double-vectorbased approach,” IEEE Trans. Ind. Electron., vol. 61, no. 11, pp. 5871-5880, Nov. 2014.

[15] P. Karamanakos, R. Mattila, and T. Geyer, "Fixed switching frequency direct model predictive control based on output current gradients," in Proc. IEEE Ind. Electron. Conf., Washington D.C., USA, 2018, pp. 23292334.

[16] T. Geyer, P. Karamanakos, and R. Kennel, "On the benefit of long-horizon direct model predictive control for drives with LC filters," in Proc. IEEE Energy Convers. Congr. Expo., Pittsburgh, PA, USA, Sept. 2014, pp. 3520-3527.

[17] S. N. Manias, Power electronics and motor drive systems. Cambridge, MA: Academic Press, 2016.

[18] J. B. Rawlings and D. Q. Mayne, Model predictive control: Theory and design. Madison, WI: Nob Hill, 2009. 